\title{
Insulin resistance and the severity of coronary artery disease based on obesity status in stable-angina patients: A cross-sectional analysis
}

\author{
Deepak Uppunda ${ }^{1}$, Ranjan Shetty ${ }^{1}$, Pragna Rao ${ }^{2}$, Abdul Razak ${ }^{1}$, Ajit Singh $^{1 *}$, Sheetal Chauhan ${ }^{3}$, Husena Vadnagarwala ${ }^{4}$, \\ Sridevi Prabhu ${ }^{5}$, Umesh Pai ${ }^{5}$ \\ ${ }^{1}$ Department of Cardiology, Kasturba Medical College, Manipal Academy of Higher Education, Manipal, India. \\ ${ }^{2}$ Department of Biochemistry, Kasturba Medical College, Manipal Academy of Higher Education, Manipal, India. \\ ${ }^{3}$ Department of Pharmacology, Melaka Manipal Medical College, Manipal Academy of Higher Education, Manipal, India. \\ ${ }^{4}$ Department of Dietetics and Applied Nutrition, Welcomgroup Graduate School of Hotel Administration, Manipal Academy of Higher Education, Manipal, \\ India. \\ ${ }^{5}$ Department of Cardiovascular Technology, School of Allied Health Sciences, Manipal Academy of Higher Education, Manipal, India.
}

\begin{tabular}{l}
\hline ARTICLE INFO \\
\hline Received on: 17/04/2019 \\
Accepted on: 21/05/2019 \\
Available online: 01/07/2019 \\
\hline Key words: \\
Metabolic obesity, \\
SYNTAX score, coronary \\
angiogram, diabetes mellitus, \\
glycosylated hemoglobin.
\end{tabular}

\begin{abstract}
The evaluation of coronary artery disease (CAD) concerning the metabolic status and body mass index (BMI) is poorly studied. This study was designed to observe the relationship between insulin resistance (IR) and the severity of CAD on the basis of the metabolic and phenotypic status in stable-angina patients. A cross-sectional study was conducted on 532 patients with stable angina and coronary angiogram was done to diagnose the CAD for all. Determination of metabolic obesity was done using the National Cholesterol Education Program-Adult Treatment Panel III criteria. Phenotypic obesity was defined as BMI $\geq 25 \mathrm{~kg} / \mathrm{m}^{2}$. Homeostasis model assessment IR in correlation with the severity of CAD was measured using SYNTAX (TAXUS Drug-Eluting Stent Versus Coronary Artery Bypass Surgery for the Treatment of Narrowed Arteries) Score. The average age of the patients was $57.58 \pm 10.40$ years, and $69.4 \%$ were males. Out of 532 subjects, $51.3 \%$ were hypertensive, $14.5 \%$ were smokers, $29.1 \%$ consumed alcohol, $49.3 \%$ were metabolically obese, and 50\% were phenotypically obese. Increase in IR increased the risk of severity of CAD in metabolically obese subjects $(\mathrm{OR}=2.51, p=0.048)$. In the phenotypically obese group, the relationship between IR and the severity of CAD was not statistically significant $(\mathrm{OR}=-2.19, p=0.08)$. The study concludes that the increased IR increases the risk of severity of CAD in metabolically obese subjects.
\end{abstract}

\section{INTRODUCTION}

Prevalence of metabolic obesity also termed as metabolic syndrome and insulin resistance (IR) is increasing drastically throughout the world, and it is most common in Asian adults. In India, $41 \%$ of the adult population with age $>21$ years are metabolically obese. Its prevalence increases with age (Ramachandran et al., 2003). The decreased ability of insulin to promote glucose utilization is called IR. The secretion and production of insulin, usually increased by pancreatic $\beta$-cells

\footnotetext{
"Corresponding Author

Ajit Singh, Department of Cardiology, Kasturba Medical College,

Manipal Academy of Higher Education, Manipal, India.

E-mail: ajitjsingh.mcops@gmail.com
}

as a compensatory mechanism (hyperinsulinemia), but in that mechanism, glucose tolerance remains normal (Oliveira et al., 2004). A problem of overall health and its effects even on children and adolescents indicates the IR. The development of obesity as seen by the occurrence of co-morbidities and complications as a result of excess weight is a big concern for the clinician (Cook et al., 2003). A set of risk factors for cardiovascular disease (CVD) is represented by metabolic obesity or metabolic syndrome. The assessment of coronary artery disease (CAD) with respect to metabolic status and body mass index (BMI) is poorly studied. The identification of metabolic obesity risk factors may be helpful in prevention of cardiovascular diseases in the near future. In patients, the development and extent of coronary CAD are not uniform. At present, IR is a known etiology for directly predicting the occurrence of CAD (Reaven, 1997), but the status of IR has not 
been investigated in depth for the severity of CAD. A significant correlation between IR and severity of CAD can be used for the detection of high-risk individuals through a simple test. Identification of patients who are not candidates for angioplasty even with severe and extensive disease can be done easily. Hence, this study aims to analyze the relationship between IR measured by homeostatic model assessment for IR (HOMA-IR) and severity of CAD measured by SYNTAX score as demonstrated via a coronary angiogram (CAG). SYNTAX score is a well-established validated tool to measure the severity of CAD in patients with diabetes mellitus (DM).

\section{METHODS AND MATERIALS USED}

\section{Study population}

The study was a prospective cross-sectional observational study conducted from January 2016 to December 2017. A total of 532 patients with stable angina who had undergone CAG for the evaluation of CAD were recruited from a tertiary care center in South India. The sample size was calculated based on the power of the study and the proportions of the groups. The patients belonged to the age group of 25-80 years. Patients with a previous history of CAD and heart failure were excluded. Those with ongoing non-cardiac comorbidities, such as infectious diseases, chronic inflammatory disorders, and viral illnesses (retroviral, hepatitis B and C) and chronic kidney disease were also excluded from the study.

\section{Ethical considerations}

The study has obtained the approval from Kasturba Medical College-Institutional Ethics Committee (IEC) of Manipal Academy of Higher Education, Manipal, India, and informed consent form was collected from all the patients. Ethical approval number was KH-IEC 644/2015.

\section{Diagnostic criteria}

All the patients underwent a personal interview using a customized questionnaire that included data on smoking and physical activity, height and weight, and waist and hip circumference were measured to calculate BMI and waisthip ratio (WHR), respectively. Anthropometric measurements were recorded before the patients underwent CAG. Details of medications, clinical history of DM, and hypertension were also recorded.

According to the NCEP-ATPIII criteria, metabolic obesity (metabolic syndrome) include five risk factors, i.e., serum Triglyceride level (>150 mg/dl), high-density lipid (HDL) levels $(<40 \mathrm{mg} / \mathrm{dl} \mathrm{male},<50 \mathrm{mg} / \mathrm{dl}$ female), plasma glucose at at least 8 hours of fasting $(>110 \mathrm{mg} / \mathrm{dl})$, blood pressure $(135 / 80 \mathrm{mmHg})$, and waist circumference ( $>102$ for male, $>88$ for female). Those patients presented with three or more of the above-mentioned risk factors were considered as metabolically obese, otherwise metabolically healthy. Based on the Asia-Pacific criteria for obesity, a BMI $>25 \mathrm{~kg} / \mathrm{m}^{2}$ in patients was considered as phenotypical obese (Weisell, 2002).

\section{Biochemical assessment}

Blood samples of the patients were collected at baseline admission (before undergoing CAG). Fasting blood sugar
(FBS), total cholesterol, and HDL cholesterol were measured using autoanalyzer, Hitachi P-800. Fasting insulin level was measured using another autoanalyzer (Roche Diagnostics, Basel, Switzerland), and sphygmomanometer was used to record the systolic blood pressure using mercury.

IR was measured by HOMA-IR computerized method, which has previously shown good correlation with a euglycemic clamp for use in cross-sectional studies. The severity of CAD was evaluated and SYNTAX Scoring method was used to calculate the same. SYNTAX Scoring was carried out by a team of cardiologists who were unaware of the other parameters recorded for each patient.

\section{Statistical analysis}

SPSS Software version 20.0 was used for statistical analysis (SPSS Inc., Chicago, Illinois). Demographic characteristics were presented using frequency and descriptive analysis. Association between the various study parameters was assessed using regression analysis (Odds ratio). Relationship between HOMA-IR and SYNTAX Score was assessed using correlation (with a 95\% confidence interval). Statistically significance was considered at a $p$-value of less than 0.05 .

\section{RESULTS}

The study population had a mean age of $57.58 \pm 10.40$ years out of which majority were males $(69.4 \%)$. Out of the total 532 subjects, $51.3 \%$ were hypertensive, $14.5 \%$ were smokers, $29.1 \%$ consumed alcohol, $49.3 \%$ were metabolically obese, and $50 \%$ were phenotypically obese. CAG was done to confirm the diagnosis of CAD and to assess the SYNTAX score. Among all, $50.5 \%$ showed mild CAD in by CAG report and rest of patients had shown to have diseased vessels and underwent percutaneous transluminal coronay angioplasty. One hundred and six (19.9\%) patients showed to have single vessel disease (SVD) followed by double vessed disease (DVD) in 77 (14.5\%) patients. Laboratory investigations related to diagnosis of the disease and to rule out comorbidities were done and mentioned with socio-demographic data as shown in Table 1.

SYNTAX score in our study was defined as low risk $(0$ $22)$ and high risk $(>22)$ and scatter plot representing the association between CAD severity score and, IR is showed in Figure 1.

IR showed a positive correlation with the severity of $\mathrm{CAD}$ in metabolically obese subjects. It indicates that an increase in IR increases the severity of CAD in metabolically obese subjects $(\mathrm{OR}=2.51, p=0.048)$ (Table 2$)$. Phenotypically obese population was not significantly correlated with IR in stable-angina patients ( $p=0.08$ with negative OR) (Table 3). Male gender is associated with high SYNTAX score, and it showed five-folds more severe heart attack in both metabolic and phenotypic obesity status $(\mathrm{OR}=5.57, p \leq 0.001$ and $\mathrm{OR}=5.13, p \leq 0.001)$.

\section{DISCUSSION}

There are studies which have shown the association of IR with CAD in relation to diabetes (Mahabala et al., 2013), but this study has assessed the relationship between IR with severity of CAD in 532 patients in relation to metabolic and phenotypic obesity. This study showed a positive statistically significant $(r=2.5, p=0.048)$ association between these parameters. 
Table 1. Socio-demographic and clinical characteristics of the study population.

\begin{tabular}{lc}
\hline Variables & Proportions $(\boldsymbol{N}=\mathbf{5 3 2})$ \\
\hline Age (mean \pm SD) & $57.58 \pm 10.40$ \\
Males & $369(69.4 \%)$ \\
Hypertension & $273(51.3 \%)$ \\
Smoking & $77(14.5 \%)$ \\
Alcohol abuse & $155(29.1 \%)$ \\
Body mass index ( $\left.\geq 25 \mathrm{~kg} / \mathrm{m}^{2}\right)$ & $266(50.0 \%)$ \\
Metabolic Obesity & $262(49.3 \%)$ \\
Mild coronary arteries disease & $269(50.5 \%)$ \\
Single vessel disease (SVD) & $106(19.9 \%)$ \\
Double vessel disease (DVD) & $77(14.5 \%)$ \\
Tripple vessel disease (TVD) & $41(7.8)$ \\
Multi vessel disease (MVD) & $35(6.6)$ \\
Waist cicumfrace (cm) & $86.1 \pm 7.21$ \\
Waist-Hip Ratio (WHR) & $0.93 \pm 0.028$ \\
FBS (mg/dl) & $103.54 \pm 18.7$ \\
Glycosylated hemoglobin (g/dl) & $5.81 \pm 0.62$ \\
Total Cholesterol (mg/dl) & $149.6 \pm 60.97$ \\
Triglycerides (mg/dl) & $125.18 \pm 50.57$ \\
High density lipids-C (mg/dl) & $45.37 \pm 12.4$ \\
Low density lipids-C (mg/dl) & $104 \pm 34.83$ \\
Total cholesterol/High density lipid ratio & $4.08 \pm 1.37$ \\
HOMA-IR & $9.42 \pm 14.76$ \\
Urea (mg/dl) & $23.9 \pm 7.98$ \\
Creatinine (mg/dl) & $0.96 \pm 0.23$ \\
Uric acid (mg/dl) & $4.36 \pm 0.94$ \\
eGFR (ml/minute/1.73 m²) & $83.3 \pm 18.6$ \\
hsCRP (mg/l) & $5.78 \pm 11.9$ \\
Hemoglobin (g/dl) & $13.65 \pm 1.96$ \\
\hline & \\
\hline &
\end{tabular}

HOMA-IR, Homeostatic model assessment for insulin resistance; eGFR; Glomerular Filtration Rate; hsCRP, High Sensitivity C-Reactive Protein.

A previously conducted study had shown that there is no association between IR with the severity of CAD in nondiabetic men (Kruszelnicka et al., 2013). Another study showed that patients with a higher level of IR had been associated with the more severe types of CAD (Graner et al., 2007).

Altered insulin signaling in endothelial cells can help in understanding an important mechanism for the increased likelihood of cardiovascular disease (Aziz and Wheatcroft, 2011). Endothelial dysfunction developed by alteration leads to the advancement of atherosclerosis along with the pro-inflammatory state generated by IR (Zornitzki et al., 2007). Although IR is known to be a part of the metabolic syndrome, the other clinical markers, such as index measurements of body-mass and truncal obesity, have low sensitivity and specificity in identifying IR (UKPDS Group, 1998).

All through the natural history of type 2 DM, IR has been the only component that has remained constant, with reference to metabolic syndrome. IR and its measurement by HOMA-IR method has remained unchanged even with the

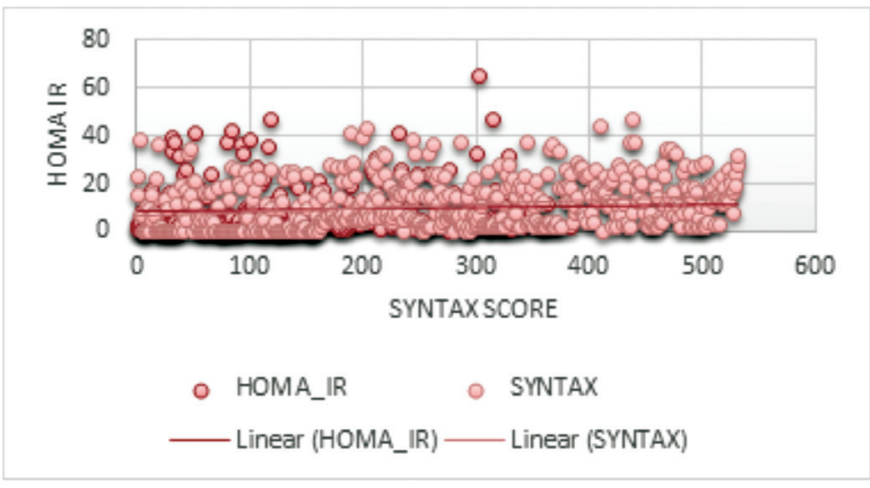

Figure 1. Scatter plot showing a positive linear correlation between the severity of CAD (SYNTAX score) and homeostasis model assessment IR in the metabolically obese group.

Table 2. Association between HOMA IR and SYNTAX score in association to metabolic status.

\begin{tabular}{lcc}
\hline SYNTAX & Coefficient & $p$-value \\
\hline Homeostatic model assessment-IR & 2.300488 & 0.142 \\
Metabolically obese & & \\
Absence & & \\
Presence & 2.511767 & 0.048 \\
Gender & & \\
Female & & \\
Male & 5.571959 & $<0.001$ \\
Smoking & & \\
Absence & & \\
Presence & -2.782323 & 0.425 \\
\hline
\end{tabular}

traditional treatment methods of type 2 DM during the many years of the UK prospective diabetes study (Wallace et al., 2004). All the other biochemical risk factors and anthropometric measurements change over a period with or without treatment. This unique feature of the IR along with its association with the severity of CAD can be used to identify the high-risk individual. A patient whose HOMA-IR is very high initially can develop severe coronary artery disease and may benefit in emergency and with specific and advanced diagnostic procedures and aggressive medical management.

Especially, in a resource-limited setting, HOMA-IR can aid the prediction of CAD and its severity with clinical relevance. Since the measurement of IR using HOMA can be performed easily, it has shown good correlation with euglycemic clamp method which is a reference standard method for measuring IR, and hence can be potentially used in routine clinical practice. Thus in the near future, we might be able to predict the severity of CAD using HOMA-IR and identification of patients who are not candidates for angioplasty even with severe and extensive disease can be done easily.

The limitation of this study is its cross-sectional design. HOMA measured IR can help us in assessing the long-term effect of risk factors in patients with CVD if it is compared with angiographic findings at the beginning of the disease. 
Table 3. Association between HOMA IR and SYNTAX score in association to obesity status.

\begin{tabular}{lcc}
\hline SYNTAX & Coefficient & $\boldsymbol{p}$ value \\
\hline Homeostatic model assessment-IR & 2.855449 & 0.068 \\
Phenotypically obese & & \\
Absence & & \\
Presence & -2.194024 & 0.08 \\
Gender & & \\
Female & & \\
Male & 5.138654 & $<0.001$ \\
\hline
\end{tabular}

\section{CONCLUSION}

According to this study, an increase in IR increases the risk of severity of CAD in metabolically obese subjects but not in the case of phenotypically obese subjects. Hence, IR can be used to predict the severity of CAD in metabolically obese subjects only.

\section{ABBREVIATIONS}

$\begin{array}{ll}\text { CVD } & \text { Cardiovascular diseases; } \\ \text { CAD } & \text { Coronary artery disease; } \\ \text { CAG } & \text { coronary angiogram; } \\ \text { IR } & \text { Insulin resistance; } \\ \text { HOMA } & \text { Homeostatic model assessment; } \\ \text { HDL } & \text { High density lipid; } \\ \text { DM } & \text { Diabetes mellitus; } \\ \text { FBS } & \text { Fasting blood sugar; } \\ \text { WHR } & \text { Waist-hip ratio; } \\ \text { BMI } & \text { Body mass index; } \\ \text { IEC } & \text { Institutional ethics committee. }\end{array}$

\section{AUTHOR'S CONTRIBUTION}

All authors have equally contributed to the research process and manuscript preparation.

\section{FINANCIAL SUPPORT}

\author{
None.
}

\section{CONFLICTS OF INTEREST}

Authors declare that there are no conflict of interest.

\section{REFERENCES}

Aziz A, Wheatcroft S. Insulin resistance in Type 2 diabetes and obesity: implications for endothelial function. Expert Rev Cardiovasc Ther, 2011; 9(4):403-7.

Cook S, Weitzman M, Auinger P, Nguyen M, Dietz W. Prevalence of a metabolic syndrome phenotype in adolescents. Arch Pediatr Adolesc Med, 2003; 157(8):821.

Graner M, Syvänne M, Kahri J, Nieminen M, Taskinen M. Insulin resistance as predictor of the angiographic severity and extent of coronary artery disease. Ann Med, 2007; 39(2):137-44.
Kruszelnicka O, Surdacki A, Golay A. Differential associations of angiographic extent and severity of coronary artery disease with asymmetric dimethylarginine but not insulin resistance in non-diabetic men with stable angina: a cross-sectional study. Cardiovasc Diabetol, 2013; 12(1):145.

Mahabala C, Srinivasan M, Manjrekar P, Unnikrishnan B, Ullal A, Kamath P, Kotekar M. Correlation of severity of coronary artery disease with insulin resistance. North Am J Med Sci, 2013; 5(10):611.

Oliveira C, Mello M, Cintra I, Fisberg M. Obesidade e síndrome metabólica na infância e adolescência. Rev Nutr, 2004; 17(2):237-45.

Ramachandran A, Snehalatha C, Satyavani K, Sivasankari S, Vijay V. Metabolic syndrome in urban Asian Indian adults - a population study using modified ATP III criteria. Diabetes Res Clin Pract, 2003; 60(3):199-204.

Reaven G. Role of insulin resistance in human disease. Nutrition, 1997; 13(1):64.

UK Prospective Diabetes Study (UKPDS) Group. Intensive blood-glucose control with sulphonylureas or insulin compared with conventional treatment and risk of complications in patients with type 2 diabetes (UKPDS 33). Lancet, 1998; 352(9131):837-53.

Wallace T, Levy J, Matthews D. Use and Abuse of HOMA Modeling. Diabetes Care, 2004; 27(6):1487-95.

Weisell R. Body mass index as an indicator of obesity. Asia Pac J Clin Nutr, 2002; 11(s8):S681-4.

Zornitzki T, Ayzenberg O, Gandelman G, Vered S, Yaskil E, Faraggi D, Caspi A, Goland S, Shvez O, Schattner A, Knobler H. Diabetes, but not the metabolic syndrome, predicts the severity and extent of coronary artery disease in women. QJM, 2007; 100(9):575-81.

\section{How to cite this article:}

Uppunda D, Shetty R, Rao P, Razak A, Singh A, Chauhan $\mathrm{S}$, Vadnagarwala $\mathrm{H}$, Prabhu S, Pai U. Insulin resistance and the severity of coronary artery disease based on obesity status in stable-angina patients: A cross-sectional analysis. J Appl Pharm Sci, 2019; 9(07):091-094. 